\title{
q-convexity properties of locally semi-proper morphisms of complex spaces
}

\author{
George-Ionut Ioniţă*
}

\begin{abstract}
We prove that if $\pi: Z \rightarrow X$ is a locally semi-proper morphism between two complex spaces and $X$ is $q$-complete, then $Z$ is $(q+r)$-complete, where $r$ is the dimension of the fiber.
\end{abstract}

\section{Introduction}

According to Grauert [11] and Narasimhan [14], [15] and their solution to the Levi problem, a complex space is Stein if and only if it admits a continuous strongly plurisubharmonic exhaustion function (see Definitions 3 and 4).

In [18], Stein showed that if $X$ and $Z$ are two complex spaces and if $\pi: Z \rightarrow X$ is an unramified covering such that $X$ is Stein, then $Z$ is Stein. This result was generalized to ramified coverings by Le Barz in [13].

The notion of a Stein space was generalized by Andreotti and Grauert in [1], where they defined $q$-convex and $q$-complete complex spaces. They extended Cartan's Theorem B and proved finiteness and vanishing theorems for the cohomology of a $q$-convex and of a $q$-complete space with values in a coherent analytic sheaf.

Also, in [3], Ballico generalized Stein's result to arbitrary q-complete spaces instead of Stein spaces and in [2], he proved the same type of result for finite morphisms of complex spaces.

\footnotetext{
${ }^{*}$ The author was supported by CNCS grant PN-II-ID-PCE-2011-3-0269.

Received by the editors in October 2013 - In revised form in September 2014.

Communicated by H. De Schepper.

2010 Mathematics Subject Classification : 32F10, 32C15.

Key words and phrases : $q$-complete space, covering space.
} 
In [5], Colţoiu and Vâjâitu considered locally trivial analytic fibrations $\pi: E \rightarrow B$ such that the fiber is a Stein curve and $B$ is $q$-complete. In this way they improved the result of [3].

Further generalizations of the results of Ballico in [2] were obtained by Vâjâitu in [19].

The purpose of this paper is to generalize the results in [2], [3], [13], [18] and [19]. This is contained in Theorem 7.

Acknowledgments: I am very grateful to Professor Mihnea Colţoiu for suggesting me this problem and for his helpful advice.

\section{Preliminaries}

All complex spaces are assumed to be reduced, finite dimensional and with countable topology.

\section{1}

Definition 1. A complex space $X$ is said to be a Stein space if the following hold:

(a) $X$ is holomorphically convex, i.e., for every compact set $K \subset X$ the holomorphically convex hull

$$
\widehat{K}_{X}=\left\{x \in X:|f(x)| \leq\|f\|_{K}, \forall f \in \mathcal{O}(X)\right\}
$$

is also compact;

(b) For every $x \in X$ there are global functions $f_{1}, \ldots, f_{N} \in \mathcal{O}(X)$ which give a local holomorphic embedding of a neighbourhood of $x$ into $\mathbb{C}^{N}$;

(c) For every pair of distinct points $x \neq y$ in $X$ there is a holomorphic function $f \in \mathcal{O}(X)$ such that $f(x) \neq f(y)$.

Definition 2. Let $D$ be an open neighbourhood of a point $z_{0} \in \mathbb{C}^{n}$ and $f \in C^{\infty}(D, \mathbb{R})$. We define the Levi form $L\left(f, z_{0}\right)$ of $f$ at $z_{0}$ as follows: for arbitrary $\xi, \eta \in \mathbb{C}^{n}$ set

$$
L\left(f, z_{0}\right)(\xi, \eta):=\sum_{i, j=1}^{n} \frac{\partial^{2} f}{\partial z_{i} \partial \bar{z}_{j}}\left(z_{0}\right) \xi_{i} \bar{\eta}_{j} .
$$

Also we set $L\left(f, z_{0}\right) \xi=L\left(f, z_{0}\right)(\xi, \xi), \xi \in \mathbb{C}^{n}$.

Definition 3. 1) A real valued $\mathcal{C}^{2}$-function $\varphi: D \rightarrow \mathbb{R}$, where $D$ is an open set in $\mathbb{C}^{n}$, is said to be plurisubharmonic (respectively strongly plurisubharmonic) if and only if its Levi form is positive-semidefinite (respectively positive definite), that is for each $z_{0} \in D$ and for every $\xi \in \mathbb{C}^{n}$ the inequality $L\left(f, z_{0}\right) \xi \geq 0$ (respectively $>0$ on $\mathbb{C}^{n} \backslash\{0\}$ ) holds.

2) Let $X$ be a complex space. A function $\varphi: X \rightarrow \mathbb{R}$ is said to be (strongly) plurisubharmonic at a point $x \in X$ if there is a local chart $\iota: U \hookrightarrow \widetilde{U} \subset \mathbb{C}^{n}$ of $X, U \ni x$ and $\widetilde{\varphi} \in C^{\infty}(\widetilde{U}, \mathbb{R})$ such that:

1. $\widetilde{\varphi} \circ \iota=\left.\varphi\right|_{U} ;$ 
2. The function $\widetilde{\varphi}$ is (strongly) plurisubharmonic on $\widetilde{U}$.

The function $\varphi$ is said to be (strongly) plurisubharmonic on a subset $W \subset X$ if it is (strongly) plurisubharmonic at every point of $W$.

Definition 4. Let $X$ be a complex space. An upper semi-continuous function $\varphi: X \rightarrow \mathbb{R}$ is said to be an exhaustion function on $X$ if the sublevel sets $\{x \in X: \varphi(x)<c\}$ are relatively compact in $X$ for any $c \in \mathbb{R}$.

We have the following result (see [11] and [14], [15]):

Theorem 1. A complex space $X$ is Stein if and only if there exists $\varphi: X \rightarrow \mathbb{R} a$ continuous strongly plurisubharmonic exhaustion function on $X$.

Definition 5. Let $X$ and $Z$ be two complex spaces. A morphism $\pi: Z \rightarrow X$ is said to be proper if for every compact set $K$ in $X$ the preimage $\pi^{-1}(K)$ is compact. A morphism $\pi: Z \rightarrow X$ is said to be finite if it is proper and it has finite fibers.

Remark 1. Let $\pi: Z \rightarrow X$ be a finite morphism of complex spaces. Then $Z$ is Stein if and only if $X$ is Stein.

We recall the following theorem of Stein [18]:

Theorem 2. Let $\pi: Z \rightarrow X$ be an unramified covering of complex spaces. If $X$ is Stein, then $\mathrm{Z}$ is Stein

Le Barz [13] extended Stein's result to locally semi-finite morphisms of complex spaces (see Definition 6 and Theorem 3).

Definition 6. Let $X$ and $Z$ be two complex spaces. We say that a morphism $\pi: Z \rightarrow X$ is

(a) semi-finite if $Z$ is the disjoint union of some open spaces $\left(W^{m}\right)_{m \in \mathbb{N}}$ such that $\left.\pi\right|_{W^{m}}: W^{m} \rightarrow X$ is a finite morphism;

(b) locally semi-finite if for all $x \in X$, there exists a neighbourhood $U \ni x$ such that $\left.\pi\right|_{\pi^{-1}(U)}: \pi^{-1}(U) \rightarrow U$ is a semi-finite morphism.

Theorem 3. Let $\pi: Z \rightarrow X$ a locally semi-finite morphism of complex spaces. If $X$ is Stein, then $\mathrm{Z}$ is Stein.

2.2 As we mentioned in the introduction, the notions of a $q$-convex and of a $q$-complete complex space were introduced in [1].

Definition 7. 1) A function $\varphi \in C^{\infty}(D, \mathbb{R})$, where $D$ is an open subset of $\mathbb{C}^{n}$ is said to be $q$-convex $(q \in \mathbb{N}, 1 \leq q \leq n)$ if its Levi form has at least $n-q+1$ positive $(>0)$ eigenvalues at every point of $U$.

2) Let $X$ be a complex space. A function $\varphi: X \rightarrow \mathbb{R}$ is said to be q-convex at a point $x \in X$ if there is a local chart $\iota: U \hookrightarrow \widetilde{U} \subset \mathbb{C}^{n}$ of $X, U \ni x$ and $\widetilde{\varphi} \in C^{\infty}(\widetilde{U}, \mathbb{R})$ such that: 
1. $\widetilde{\varphi} \circ \iota=\left.\varphi\right|_{U ;}$

2. The function $\widetilde{\varphi}$ is q-convex on $\widetilde{U}$.

The second condition can be replaced by the following:

2 '. There exists a complex linear space $E \subset \mathbb{C}^{n}, \operatorname{dim} E \geq n-q+1$ such that the Levi form $L(\widetilde{\varphi}, \iota(x))$ is positive definite when restricted to $E$.

The function $\varphi$ is said to be $q$-convex on a subset $W \subset X$ if it is $q$-convex at every point of $W$.

Definition 8. A complex space $X$ is said to be $q$-convex, if there exists a compact subset $K$ of $X$ and a smooth exhaustion function $\varphi: X \rightarrow \mathbb{R}$, which is $q$-convex on $X \backslash K$. If we can choose $K=\varnothing$, then $X$ is said to be q-complete.

Remark 2. From [14] and [15] we have that a complex space X is Stein if and only if is 1-complete.

Ballico [3] improved Theorem 2 in another direction.

Theorem 4. Let $\pi: Z \rightarrow X$ be an unramified covering. If $X$ is q-complete, then $Z$ is q-complete.

Also, in [2], Ballico showed that if $\pi: Z \rightarrow X$ is a finite morphism between complex spaces and $X$ is $q$-complete or $q$-convex, then $Z$ is $q$-complete or $q$-convex, respectively.

Colţoiu and Vâjâitu [5] proved that if $\pi: E \rightarrow B$ is a locally analytic fibration of complex spaces such that the fiber is a Stein curve and $B$ is $q$-complete, then $E$ is $q$-complete. The case when $E$ is a topological covering of $B$ was already done in [3].

Vâjâitu [19] generalized Ballico's results from [2] and showed the following:

Theorem 5. Let $\pi: Z \rightarrow X$ be a proper holomorphic map between finite dimensional complex spaces. If $X$ is $q$-complete, then $Z$ is $(q+r)$-complete, where $r$ is the dimension of the fiber.

Let $X$ be a complex space of complex dimension $n$ and $q$ an integer with $1 \leq q \leq n$. For $q>1$ the sum and the maximum of two $q$-convex functions on $X$ is not $q$-convex as they might have different directions of positivity. It was proved in [7] and [8] that every $q$-convex function with corners (i.e., a function which locally is equal to the maximum of a finite family of $q$-convex functions) can be approximated by a $\widetilde{q}$-convex function, where $\widetilde{q}=n-\left[\frac{n}{q}\right]+1$ (here $\left[\frac{n}{q}\right]$ denotes as usual the largest integer $\leq \frac{n}{q}$ ). Diederich and Fornaess also showed that this $\widetilde{q}$ is optimal. As a consequence, a finite intersection of $q$-convex open sets is $\widetilde{q}$-convex. The optimality of this $\widetilde{q}$ is proved by Chiriacescu, Colţoiu and Joiţa in [4] in the case of quasi-projective varieties in a cohomological context.

To overcome this type of problem, M. Peternell [16] introduced the notion of convexity with respect to a linear set $\mathcal{M}$. 
As before $X$ is a reduced, finite dimensional and with countable topology complex space. For any $x \in X$ we denote by $T_{x} X$ the Zariski tangent space of $X$ to $x$. Set $T X=\bigcup_{x \in X} T_{x} X$. Consider an arbitrary subset $\mathcal{M} \subset T X$ and for any point $x \in X$ put $\mathcal{M}_{x}=\mathcal{M} \cap T_{x} X$. Then $\mathcal{M}$ is said to be a linear set over $X$ if $\mathcal{M}_{x}$ is a complex vector subspace of $T_{x} X$ for any $x \in X$.

Let now $\Omega \subset X$ be an open subset. We define:

(i) $\operatorname{codim}_{\Omega} \mathcal{M}=\sup _{x \in \Omega} \operatorname{codim} \mathcal{M}_{x}$;

(ii) $\left.\mathcal{M}\right|_{\Omega}$ as $\left(\left.\mathcal{M}\right|_{\Omega}\right)_{x}=\mathcal{M}_{x}$ for every $x \in \Omega$.

Let $\pi: Y \rightarrow X$ be an analytic morphism of complex spaces and $\mathcal{M}$ a linear set over $X$. For every $y \in Y$ we have the tangent map which is a $\mathbb{C}$-linear map of complex vector spaces $\pi_{*, y}: T_{y} Y \rightarrow T_{x} X$, where $x=\pi(y)$. We set

$$
\pi^{*} \mathcal{M}:=\bigcup_{y \in Y}\left(\pi_{*, y}\right)^{-1}\left(\mathcal{M}_{x}\right)
$$

We have that $\pi^{*} \mathcal{M}$ is a linear set over $Y$. Moreover, if codim $\mathcal{M} \leq q-1$, then codim $\pi^{*} \mathcal{M} \leq q-1$.

The following are due to M. Peternell (see [16]).

Definition 9. Let $X$ be a complex space, $W \subset X$ an open subset, $\mathcal{M}$ a linear set over $W$ and $\varphi: W \rightarrow \mathbb{R}$ a smooth function.

(a) Let $x \in W$. Then $\varphi$ is said to be weakly 1 -convex with respect to $\mathcal{M}_{x}$ if there are a local chart $\iota: U \hookrightarrow \widetilde{U}$ of $X$ with $x \in U \subset W$ and $\widetilde{\varphi} \in \mathcal{C}^{\infty}(\widetilde{U}, \mathbb{R})$ such that

$$
\widetilde{\varphi} \circ \iota=\left.\varphi\right|_{U} \text { and } L(\widetilde{\varphi}, \iota(x)) \iota_{*} \xi \geq 0 \text { for any } \xi \in \mathcal{M}_{x}
$$

Furthermore, $\varphi$ is said to be weakly 1-convex with respect to $\mathcal{M}$ if $\varphi$ is weakly 1-convex with respect to $\mathcal{M}_{x}$ for every $x \in W$.

(b) We say that $\varphi$ is 1-convex with respect to $\mathcal{M}$, if for any $x \in W$ there exist an open neighbourhood $U \subset W$ of $x$ and a 1-convex function $\psi \in \mathcal{C}^{\infty}(U, \mathbb{R})$ such that $\left.\varphi\right|_{U}-\psi$ is weakly 1-convex with respect to $\left.\mathcal{M}\right|_{U}$.

Definition 10. Let $X$ be a complex space and $\mathcal{M}$ a linear set over $X$. We denote by $\mathcal{B}(X, \mathcal{M})$ the set of all continuous functions $\varphi: X \rightarrow \mathbb{R}$ such that every point of $X$ admits an open neighbourhood $D$ on which there are functions $f_{1}, \ldots, f_{k} \in \mathcal{C}^{\infty}(D, \mathbb{R})$ which are 1-convex with respect to $\left.\mathcal{M}\right|_{D}$ and

$$
\left.\varphi\right|_{D}=\max \left(f_{1}, \ldots, f_{k}\right) .
$$

We need also the following results of M. Peternell (see [16]):

Lemma 1. Suppose that $\varphi$ is a q-convex function on a complex space $X$. Then there exists a linear set $\mathcal{M}$ over X of codimension $\leq q-1$ such that $\varphi$ is 1 -convex with respect to $\mathcal{M}$. 
Lemma 2. Let $\iota: U \hookrightarrow \widetilde{U}$ be a local chart of the complex space $X$ and $\varphi: U \rightarrow \mathbb{R}$ a smooth function. Then $\varphi$ is 1-convex with respect to some linear set $\mathcal{M}$ if and only if for every compact subset $K \subset U$ there exists $\delta>0$ and for each $x \in K$ there exist $\widetilde{\varphi} \in C^{\infty}(\widetilde{U}, \mathbb{R})$ such that $\widetilde{\varphi} \circ \iota=\varphi$ and

$$
L(\widetilde{\varphi}, \iota(x)) \iota_{*}(\xi) \geq \delta\left\|\iota_{*}(\xi)\right\|^{2}
$$

for all $\xi \in \mathcal{M}_{x}$.

In general, an increasing union of Stein open subsets $\left\{X_{i}\right\}_{i \in \mathbb{N}}$ of a complex space $X$ is not Stein, even if $X$ is smooth (see [9] and [10]). However, if $\left(X_{i+1}, X_{i}\right)$ is Runge, then $\bigcup_{i \in \mathbb{N}} X_{i}$ is Stein. We recall that if $Y$ is a Stein open subset of a Stein space $X$, then $(X, Y)$ is said to be a Runge pair if for any compact subset $K$ of $Y$, the set $\widehat{K}_{X} \cap Y$ is compact. Using the approximation theorem of Oka-Weil, $(X, Y)$ is a Runge pair if and only if $Y$ is a Stein space and every holomorphic function on $Y$ can be approximated uniformly on compact subset of $Y$ by holomorphic functions on $X$.

The following result follows from Theorem 3 proved by Colţoiu and Vâjâ-itu in [6]; it gives us a criterion for testing the $q$-completeness of a complex space. The same kind of result as Theorem 3 in [6] was obtained in the $q$-concave case in [12].

Theorem 6. Let $X$ be a complex space and $\mathcal{M}$ a linear set over $X$. Let $\left\{X_{i}\right\}_{i \in \mathbb{N}}$ be an increasing sequence of open subsets of $X$ such that $X=\bigcup_{i \in \mathbb{N}} X_{i}$ and there are functions $u_{i}: X_{i} \rightarrow \mathbb{R}, u_{i} \in \mathcal{B}\left(X_{i},\left.\mathcal{M}\right|_{X_{i}}\right)$ and constants $C_{i}, D_{i} \in \mathbb{R}, C_{i}<D_{i}, i \in \mathbb{N}$ with the following properties:

(a) $\left\{x \in X_{i}: u_{i}(x)<D_{i}\right\} \subset \subset X_{i}$ for every $i \in \mathbb{N}$

(b) $\left\{x \in X_{i+1}: u_{i+1}(x)<C_{i}\right\} \subset\left\{x \in X_{i}: u_{i}(x)<D_{i}\right\}$ for every $i \in \mathbb{N}$;

(c) for every compact set $K \subset X$ there is $j=j(K) \in \mathbb{N}$ such that

$$
K \subset\left\{x \in X_{i+1}: u_{i+1}(x)<C_{i}\right\} \text { for every } i \geq j .
$$

Then there exists an exhaustion function $v \in \mathcal{B}(X, \mathcal{M})$. In particular, if codim $\mathcal{M} \leq$ $q-1$, then $X$ is q-complete.

2.3 Let $X$ be a complex space and $A$ an analytic subset of $X$. The Andreotti function will help us to get some positive eigenvalues in the "normal direction" at the

regular points of $A$. Denote by $\mathfrak{I}_{A}$ the coherent ideal sheaf of germs of holomorphic functions vanishing along $A$.

Choose a locally finite covering $\left\{U_{j}\right\}_{j}$ of $X$ by relatively compact open subsets of $X$ such that on each $U_{j}$ there are functions $h_{1}^{(j)}, \ldots, h_{q(j)}^{(j)} \in \mathcal{O}\left(U_{j}\right)$ with $\mathfrak{I}_{A} \mid u_{j}=$ $\left(h_{1}^{(j)}, \ldots, h_{q(j)}^{(j)}\right)$. 
Let $\left\{\rho_{j}\right\}_{j}$ be a partition of unity subordinated to the covering $\left\{U_{j}\right\}_{j}$ of $X$.

We define the Andreotti function $f_{A}: X \rightarrow \mathbb{R}$ by setting:

$$
f_{A}(x)=\sum_{j} \rho_{j}(x)\left\|h^{(j)}(x)\right\|^{2}, x \in X,
$$

where

$$
\left\|h^{(j)}\right\|^{2}=\sum_{i=1}^{q(j)}\left|h_{i}^{(j)}\right|^{2} .
$$

We remark that $f_{A} \geq 0, f_{A} \in C^{\infty}(X)$ and $A=\left\{f_{A}=0\right\}$.

Suppose that $x_{0} \in A$ and let $\iota: U \hookrightarrow \widetilde{U}$ be a local chart around $x_{0}$. Extend $\rho_{j}$ by $\widetilde{\rho}_{j} \in C_{0}^{\infty}(\widetilde{U})$ and $\left.h_{i}^{(j)}\right|_{U}$ by $\widetilde{h}_{i}^{(j)} \in \mathcal{O}(\widetilde{U})$. Locally, $f_{A}$ has an extension $\widetilde{f}_{A}$ defined on $\widetilde{U}$ such that

1. the Levi form $L\left(\widetilde{f}_{A}, \iota(x)\right)$ is positive semidefinite for all $x \in U \cap A$;

2. for $x \in U \cap \operatorname{Reg}(A)$ we have that the Levi form $L\left(\widetilde{f}_{A}, \iota(x)\right)(v)=0$ iff $v \in$ $\iota_{*, x}\left(T_{x} A\right)$.

The Andreotti function is used in the next result which follows from Lemma 3 and Lemma 4 in [19]:

Lemma 3. Let $\pi: Z \rightarrow X$ be a holomorphic map between finite dimensional reduced complex spaces and $A$ an analytic subset of $Z$. Put $r=\max \left\{\operatorname{dim} \pi^{-1}(x): x \in X\right\}$ and let $B \subset A$ be an analytic subset such that Sing $(A) \subseteq B$ and suppose that the restriction map $\left.\pi\right|_{A \backslash B}: A \backslash B \rightarrow X$ has locally constant rank.

Assume also that there exists a locally finite covering $\left\{V_{l}^{\prime}\right\}_{l}$ of $X$ by relatively compact open subsets and 1-convex functions $\varphi_{l}: V_{l}^{\prime} \rightarrow \mathbb{R}_{+}$. Let $V_{l} \subset V_{l}^{\prime}$ be open subsets, $\bar{V}_{l} \subset V_{l}^{\prime}$ and $\cup V_{l}=X$. Denote $U_{l}:=\pi^{-1}\left(V_{l}\right), U_{l}^{\prime}:=\pi^{-1}\left(V_{l}^{\prime}\right)$ and put

$$
\psi_{l}=f_{A}+\varphi_{l} \circ \pi: U_{l}^{\prime} \rightarrow \mathbb{R}_{+}
$$

where $f_{A}$ is the Andreotti function of the analytic subset $A$ of $Z$.

Then there is an open neighbourhood $\Omega$ of $A \backslash B$ in $Z$ and a linear set $\mathcal{M}$ over $\Omega$, codim $\mathcal{M} \leq r$ such that $\left.\psi_{l}\right|_{U_{l} \cap \Omega}$ is 1-convex with respect to $\left.\mathcal{M}\right|_{U_{l} \cap \Omega}$ for any $l$.

\section{The main result}

Following the ideas of Le Barz [13] we give the next definition.

Definition 11. Let $X$ and $Z$ be two complex spaces. We say that a morphism $\pi: Z \rightarrow X$ is

(a) semi-proper if $Z$ is the disjoint union of some open spaces $\left(W^{m}\right)_{m \in \mathbb{N}}$ such that $\left.\pi\right|_{W^{m}}: W^{m} \longrightarrow X$ is proper;

(b) locally semi-proper if for all $x \in X$, there exists a neighbourhood $U \ni x$ such that $\left.\pi\right|_{\pi^{-1}(U)}: \pi^{-1}(U) \longrightarrow U$ is a semi-proper morphism. 
Now we are ready to state the main result.

Theorem 7. Let $X$ and $Z$ be two complex spaces and $\pi: Z \rightarrow X$ a locally semiproper morphism and $r=\max \left\{\operatorname{dim} \pi^{-1}(x): x \in X\right\}$. If $X$ is q-complete, then $Z$ is $(q+r)$-complete.

Proof. Since $X$ is $q$-complete there exists a smooth $q$-convex exhaustion function $\varphi: X \rightarrow \mathbb{R}$ on $X$. Due to Lemma 1 there exists a linear set $\mathcal{M}$ over $X$ of codimension $\leq q-1$ such that $\varphi$ is 1 -convex with respect to $\mathcal{M}$. The idea is to use the $q$-completeness criterion provided by Theorem 6 .

Now we need the following result from [19]:

Proposition 1. Let $\pi: Z \rightarrow X$ be a holomorphic map. Then there exists a decreasing chain of $p+1$ analytic subsets $A_{k}$ of $Z$, where $p \leq \operatorname{dim} Z, Z=A_{p} \supset A_{p-1} \supset \cdots \supset$ $A_{1} \supset A_{0}=\varnothing$ such that for every $k \in\{1,2, \ldots, p\}$ we have $\operatorname{dim} A_{k-1}<\operatorname{dim} A_{k}$, Sing $\left(A_{k}\right) \subset A_{k-1}$ and

$$
\left.\pi\right|_{A_{k} \backslash A_{k-1}}: A_{k} \backslash A_{k-1} \rightarrow X
$$

has locally constant rank.

The above decomposition of $Z$ with respect to $\pi$ is called the singular filtration of $\pi$ (see also [17]).

So, consider $A_{1} \supset A_{2} \supset \cdots \supset A_{p}$ the analytic subsets of $Z$ given by Proposition 1 and the corresponding Andreotti functions $f_{A_{k}}, k=\overline{1, p}$.

The next ingredient that we need is a lemma. This lemma was proved by Le Barz [13] in the case of 0-dimensional fibers, but the proof in the general case (the dimension of the fiber is $>0$ ) goes exactly the same way.

Lemma 4. Let $X$ and $Z$ be two complex spaces and $\pi: Z \rightarrow X$ a locally semi-proper morphism. Then there exists a locally finite covering $\left\{U_{j}\right\}_{j}$ of $Z$ and a locally finite covering $\left\{V_{l}\right\}_{l}$ of $X$ such that the following conditions hold:

1. for all $j$, there exists a positive integer $m_{j}$ and a local chart $\iota_{j}: U_{j} \hookrightarrow \widetilde{U}_{j}$, where $\widetilde{U}_{j}$ is an open subset of $\mathbb{C}^{m_{j}}$;

2. for all $l$, there exists a positive integer $n_{l}$ and a local chart $\tau_{l}: V_{l} \hookrightarrow \widetilde{V}_{l}$, where $\widetilde{V}_{l}$ is an open subset of $\mathbb{C}^{n_{l}}$;

3. for all $j$, there exists $l(j)$ such that we have $\pi\left(U_{j}\right) \subset V_{l(j)}$ and $\left.\pi\right|_{U_{j}}$ extends to a holomorphic map $\widetilde{\pi}: \widetilde{U}_{j} \rightarrow \widetilde{V}_{l(j)}$;

Also there exists a $C^{\infty}$ function $f: Z \rightarrow \mathbb{R}$ such that:

- $\left\{z \in Z: f(z)<c_{1}\right\} \cap\left\{z \in Z:(\varphi \circ \pi)(z)<c_{2}\right\} \subset \subset Z, \forall c_{1}, c_{2} \in \mathbb{R}$;

- for all $j$, there exists a map $g_{j}: V_{l(j)} \rightarrow \mathbb{R}$ such that $\left.f\right|_{U_{j}}=\left.g_{j} \circ \pi\right|_{U_{j}}$;

- $g_{j}$ has a $C^{\infty}$ extension, $\widetilde{g}_{j}: \widetilde{V}_{l(j)} \rightarrow \mathbb{R}$; 
- for all compact sets $K \subset X$,

$$
\sup _{j \in \mathbb{N}}\left\{\left|\frac{\partial^{2} \widetilde{g}_{j}}{\partial z_{r}^{(l(j))} \partial \bar{z}_{s}^{(l(j))}}\right|_{\left.\right|_{\tau_{l(j)}\left(V_{l(j)} \cap K\right)}}: V_{l(j)} \cap K \neq \varnothing, r, s=\overline{1, n_{l(j)}}\right\}<\infty .
$$

We choose $\left\{W_{j}^{1}\right\}_{j}$ a locally finite covering of $Z$ and $\left\{W_{k}^{2}\right\}_{k}$ a locally finite covering of $X$ such that the conditions in Lemma 4 hold. We denote by $\widetilde{\varphi}: \widetilde{W}_{k(j)}^{2} \rightarrow \mathbb{R}$ a $q$-convex extension of $\left.\varphi\right|_{W_{k(j)}^{2}}$. Also consider the function $f: Z \rightarrow \mathbb{R}$, the function $g_{j}: W_{k(j)}^{2} \rightarrow \mathbb{R}$ and its extension $\widetilde{g}_{j}$ given by Lemma 4 .

Using the boundedness condition for the second derivatives of the function $f$, on every compact set, there exists a convex and strictly increasing function $\chi$ so that $\left.\left(\chi \circ \widetilde{\varphi}+\widetilde{g}_{j}\right)\right|_{\widetilde{W}_{k(j)}^{2}}$ is $q$-convex for all $j$.

Because $\left\{z \in Z: f(z)<c_{1}\right\} \cap\left\{z \in Z:(\varphi \circ \pi)(z)<c_{2}\right\} \subset \subset Z, \forall c_{1}, c_{2} \in \mathbb{R}$ we get that $\chi \circ \varphi \circ \pi+f$ is an exhaustion function. We denote by $Z_{i}$ the sublevel sets $\{\chi \circ \varphi \circ \pi+f<i\}$ which are relatively compact in $Z$. This increasing sequence of open sets that cover $Z$ will be the one that is needed in Theorem 6 .

Now we have to build the functions $u_{i}$ in Theorem 6 . For this we will make use of a lemma which is based upon Lemma 3. For details one should consult the Main Lemma of [19] and the Remark that follows.

Lemma 5. Let $\pi: Z \rightarrow X$ be a holomorphic map between reduced complex spaces with $r=\max \left\{\operatorname{dim} \pi^{-1}(x): x \in X\right\}$. Then there exists $\mathcal{N}$ a linear set of codimension $\leq r$ over $Z$ such that for any relatively compact open subset $U$ of $Z$, there exists a finite covering $\left\{V_{l}\right\}_{l}$ of $\overline{\pi(U)}$ by relatively compact open subsets and smooth functions $\psi_{l}: U_{l} \rightarrow \mathbb{R}_{+}$such that $\psi_{l}$ is 1 -convex with respect to $\mathcal{N}$ over $U_{l} \cap U$, where $U_{l}=\pi^{-1}\left(V_{l}\right)$.

Now we go back to the proof. Since $Z_{i} \subset \subset Z$, there exists a linear set $\mathcal{N}$ of codimension $\leq r$ over $Z$, a finite covering $\left\{V_{l}^{i}\right\}_{l}$ of $\overline{\pi\left(Z_{i}\right)}$ by relatively compact open subsets and smooth functions $\psi_{l}^{i}: U_{l}^{i} \rightarrow \mathbb{R}_{+}$such that $\psi_{l}^{i}$ is 1-convex with respect to $\mathcal{N}$ over $U_{l}^{i} \cap Z_{i}$, where $U_{l}^{i}=\pi^{-1}\left(V_{l}^{i}\right)$. The functions $\psi_{l}^{i}$ may be taken $>0$.

Let $\left\{\rho_{l}^{i}\right\}_{l}$ be a partition of unity subordinated to the covering $\left\{V_{l}^{i}\right\}_{l}$ and we define a smooth function $u_{i}$ on $Z_{i}$ as follows:

$$
u_{i}=\chi \circ \varphi \circ \pi+f+\sum_{l} \epsilon_{l}^{i} \cdot\left(\rho_{l}^{i} \circ \pi\right)^{2} \cdot \psi_{l}^{i}
$$

where $\epsilon_{l}^{i}>0$ are sufficiently small constants to be chosen later in the proof. Since the above sum is $>0$, there exists $\delta_{i}>0$ such that for all $z \in Z_{i}$ we have $\sum \epsilon_{l}^{i}$. $\left(\rho_{l}^{i} \circ \pi\right)^{2} \cdot \psi_{l}^{i} \geq \delta_{i}$. By choosing the constants $\epsilon_{l}^{i}$ to be sufficiently small we may assume that $\sum \epsilon_{l}^{i} \cdot\left(\rho_{l}^{i} \circ \pi\right)^{2} \cdot \psi_{l}^{i}<1$.

First we show that the functions $u_{i}$ satisfy the conditions (a), (b) and (c) from Theorem 6. We define $C_{i}:=i-1$ and $D_{i}:=i$. For simplicity we denote $\sum \epsilon_{l}^{i} \cdot\left(\rho_{l}^{i} \circ \pi\right)^{2} \cdot \psi_{l}^{i}$ by $\sum^{i}$. Since $\sum^{i} \geq \delta_{i}$, we have that $\left\{u_{i}<i\right\} \subset \subset\{\chi \circ \varphi \circ \pi+f<$ 
$i\}$, so this proves (a). For the second condition, let $z \in Z_{i+1}$ such that $u_{i+1}(z)<$ $i-1$. We get that $\chi \circ \varphi \circ \pi+f+\sum^{i+1}<i-1$, thus $\chi \circ \varphi \circ \pi+f<i-1$ and $z \in Z_{i}$. Adding $\sum^{i}$ to the last inequality, we obtain $\chi \circ \varphi \circ \pi+f+\sum^{i}<i-1+$ $\sum^{i}<i$, since $\sum^{i}<1$. Now for condition (c), since $\bigcup\{\chi \circ \varphi \circ \pi+f<i-2\}=Z$, it is enough to prove that $\left\{z \in Z_{i+1}: \chi \circ \varphi \circ \pi+f<i-2\right\} \subset\left\{z \in Z_{i+1}: u_{i+1}<\right.$ $i-1\}$. Adding $\Sigma^{i}$ to $\chi \circ \varphi \circ \pi+f$ and using the fact that $\sum^{i}<1$, we easily get the claim.

Now we prove that $u_{i} \in \mathcal{B}\left(Z_{i},\left.\mathcal{P}\right|_{Z_{i}}\right)$, where $\mathcal{P}:=\pi^{*} \mathcal{M} \cap \mathcal{N}$. We have that $\mathcal{P}$ is a linear set over $Z$ and codim $\mathcal{P} \leq q+r-1$. It is enough to show that every point $z \in Z_{i}$ admits an open neighbourhood $D$ such that $u_{i}$ is 1-convex with respect to $\left.\mathcal{P}\right|_{D}$. Using Lemma 2, this is equivalent to proving that for every compact $K \subset Z_{i}$ there exists $\delta>0$ and for all $z \in K$ there exists an extension $\widetilde{u}_{i}$ of $u_{i}$ such that

$$
L\left(\widetilde{u}_{i}, \iota(z)\right) \iota_{*}(\xi) \geq \delta\left\|\iota_{*}(\xi)\right\|^{2}
$$

for all $\xi \in \mathcal{P}_{z}$.

This is a local statement. So, without any loss of generality, we may suppose that there are local charts $\iota: U \hookrightarrow \widetilde{U} \subset \mathbb{C}^{m}, z \in U \subset Z_{i}, K \subset U$ and $\tau: V \hookrightarrow \widetilde{V} \subset$ $\mathbb{C}^{n}, x:=\pi(z) \in V \subset X, \pi(K) \subset V$ such that:

(i) $\pi(U) \subset V$ and there exists an extension $\widetilde{\pi}: \widetilde{U} \rightarrow \widetilde{V}, \tilde{\pi} \circ \iota=\tau \circ\left(\left.\pi\right|_{U}\right)$;

(ii) there exists $A>0$ and smooth extensions $\widetilde{\varphi}: \widetilde{V} \rightarrow \mathbb{R}_{+}$and $\widetilde{g}: \widetilde{V} \rightarrow \mathbb{R}_{+}$ such that

$$
L(\chi \circ \widetilde{\varphi}+\widetilde{g}, \tau(x)) \tau_{*}(\zeta) \geq A\left\|\tau_{*}(\zeta)\right\|^{2}
$$

for all $\zeta \in \mathcal{M}_{x}$ and $x \in \pi(K)$ (this is true due to Lemma 4);

(iii) there are constants $a_{l}>0$ and smooth extensions $\widetilde{\psi}_{l}: \widetilde{U} \rightarrow \mathbb{R}_{+}$of $\psi_{l}$ such that

$$
L\left(\widetilde{\psi}_{l}, \iota(z)\right) \iota_{*}(\widetilde{\zeta}) \geq a_{l}\left\|\iota_{*}(\xi)\right\|^{2}
$$

for all $\xi \in \mathcal{N}_{z}$ and $z \in K$ (this is true due to Lemma 5).

Let $\widetilde{\rho}_{l}$ be smooth extensions of $\rho_{l}$ to $\widetilde{V}$.

So we get an extension $\widetilde{u}_{i}: \widetilde{U} \rightarrow \mathbb{R}_{+}$of $\left.u_{i}\right|_{U}$ given as follows:

$$
\widetilde{u}_{i}=\chi \circ \widetilde{\varphi} \circ \widetilde{\pi}+\widetilde{g} \circ \widetilde{\pi}+\sum_{l} \epsilon_{l} \cdot\left(\widetilde{\rho}_{l} \circ \widetilde{\pi}\right)^{2} \cdot \widetilde{\psi}_{l} .
$$

Now, using the same computations as in [19] (see Theorem A, pages 231-232), we get, for a sufficiently small positive $\epsilon$, that for any choice of the constants $\epsilon_{l}$, with $0<\epsilon_{l} \leq \epsilon$, the Levi form of $\widetilde{u}_{i}$ at $\iota(z)$ in direction $l_{*}(\xi)$ is strictly positive for $\xi \in \mathcal{P}_{z}$. This means that there exists $\delta>0$ such that $L\left(\widetilde{u}_{i}, \iota(z)\right) \iota_{*}(\xi) \geq \delta\left\|\iota_{*}(\xi)\right\|^{2}$ for all $\xi \in \mathcal{P}_{z}$. 


\section{References}

[1] Andreotti, A., Grauert, H.: Théorèmes de finitude pour la cohomologie des espaces complexes, Bulletin de la S.M.F. 90 (1962), 193-259.

[2] Ballico, E.: Morfismi finiti tra spazi complessi e q-convessità, Ann. Univ. Ferrara, sez. VII - Sc. Mat. 26 (1980), 29-31.

[3] Ballico, E.: Rivestimenti di spazi complessi e q-completezza, Riv. Mat. Univ. Parma 7 (1981), 443-452.

[4] Chiriacescu, G., Colţoiu, M., Joiţa, C.: Analytic cohomology groups in top degrees of Zariski open sets in $\mathbb{P}^{n}$, Math. Z. 264 (2010), 671-677.

[5] Colţoiu, M., Vâjâitu, V.: Locally trivial fibrations with singular 1-dimensional Stein fiber over q-complete spaces, Nagoya Math. J. 157 (2000), 1-13.

[6] Colţoiu, M., Vâjâitu, V.: On the n-completeness of covering spaces with parameters, Math. Z. 237 (2001), 815-831.

[7] Diederich, K., Fornaess, J. E.: Smoothing q-convex functions and vanishing theorems, Invent. Math. 82 (1985), 291-305.

[8] Diederich, K., Fornaess, J. E.: Smoothing q-Convex Functions in the Singular Case, Math. Ann. 273 (1986), 665-671.

[9] Fornaess, J. E.: An Increasing Sequence of Stein Manifolds whose Limit is not Stein, Math. Ann. 223 (1976), 275-277.

[10] Fornaess, J. E.: 2 Dimensional Counterexamples to Generalizations of the Levi Problem, Math. Ann. 230 (1977), 169-173.

[11] Grauert, H.: On Levi's Problem and the Imbedding of Real Analytic Manifolds, Ann. Math. 68 (1958), 460-472.

[12] Joiţa, C.: On the n-concavity of covering spaces with parameters, Math. Z. 245 (2003), 221-231.

[13] Le Barz, P.: A propos des revêtements ramifiés d'espaces de Stein, Math. Ann. 222 (1976), 63-69.

[14] Narasimhan, R.: The Levi Problem for Complex Spaces, Math. Ann. 129 (1961), 355-365.

[15] Narasimhan, R.: The Levi Problem for Complex Spaces II, Math. Ann. 146 (1962), 195-216.

[16] Peternell, M.: Algebraische Varietäten und q-vollständige komplexe Räume, Math. Z. 200 (1989), 547-581.

[17] Sommese, A. J.: A convexity theorem, Proc. Symp. Pure Math. 40 Part 2 (1983), 497-505. 
[18] Stein, K.: Überlagerung holomorph vollständiger komplexer Räume, Arch. Mat. 7 (1956), 354-361.

[19] Vâjâitu, V.: Some convexity properties of morphisms of complex spaces, Math. Z. 217 (1994), 215-245.

Simion Stoilow Institute of Mathematics of the Romanian Academy

Research group of the project ID-3-0269

P.O. Box 1-764, Bucharest 014700, Romania

and

Department of Mathematics and Computer Science,

"Politehnica" University of Bucharest,

313 Splaiul Independentei,

Bucharest 060042, Romania.

E-mail address: georgeionutionita@yahoo.com 